\title{
Interest of the six-minute walk test in the functional assessment of former pulmonary tuberculosis patients
}

\section{Intérêt $d u$ test de marche de six minutes dans l'évaluation fonctionnelle des anciens tuberculeux pulmonaires}

\author{
AR. Ouédraogo ${ }^{1,2}$, AS. Adambounou ${ }^{2}$, \\ K. Aziagbé2 ${ }^{2}$ K. Adjoh', M. Ouédraogo ${ }^{1}$ \\ 1: Service de Pneumologie. CHU Yalgado Ouedraogo. Ouagadougou, Burkina Faso \\ 2: Service de Pneumologie. CHU Sylvanus Olympio. Lomé, Togo \\ 3: Centre Hospitalier Universitaire Regional de Ouahigouya. Burkina Faso \\ 4: Centre Hospitalier Universitaire de Kara. Togo
}

\begin{abstract}
Introduction. Pulmonary tuberculosis (PT) can provide many sequels often very disabling. The purpose of this work was to evaluate sub-maximal exercise tolerance in former bacteriologically confirmed, treated, and cured TB patients.

Patients and methods. This was a cross-sectional study of data collected from treated and cured pulmonary TB patients in Lomé, Togo. The 6-minute walk test (TM6) was used for the sub-maximal exercise tolerance assessment and spirometry for the detection of ventilatory disorders (TV)

Results. A total of 113 patients were included. The average age was $33.95 \pm 10.3$ years. Men accounted for $69.9 \%$ of the workforce. The average distance traveled at TM6 was $635.55 \pm 89.38$ meters with extremes of 441 and 849 meters. Fifteen percent of our patients had traveled less than normal. There was a statistically significant association between female gender $(p=0.005)$, diagnostic delay $(p=0.006)$ and abnormal distances traveled. In addition, $67.3 \%$ of patients had a spirometry TV dominated by the restrictive syndrome (81.58\%). Compared with spirometry, TM6 was sensitive in $78.95 \%$ of cases and specific in $2.7 \% \%$.

Conclusion. PT can significantly alter the respiratory function of patients. TM6 can be an instrument for the functional assessment of PT sequelae.
\end{abstract}

KEYWORDS: Tuberculosis; Walking test; Spirometry.

\section{RÉSUMÉ}

Introduction. La tuberculose pulmonaire $(\mathrm{TP})$ peut être pourvoyeuse de nombreuses séquelles souvent très handicapantes. Le but de ce travail était d'évaluer la tolérance à l'effort sous-maximal chez les anciens patients tuberculeux bactériologiquement confirmés, traités et déclarés guéris.

Patients et méthode. Il s'est agi d'une étude transversale, les données ont été recueillies auprès des tuberculeux pulmonaires traités et déclarés guéris, à Lomé au Togo. Le test de marche de 6 minutes (TM6) a servi pour l'évaluation de la tolérance à l'effort sous-maximal et la spirométrie pour la détection des troubles ventilatoires (TV).

Résultats. Un total de 113 patients a été inclus. La moyenne d'âge était de 33,95 $\pm 10,3$ ans. Les hommes représentaient $69,9 \%$ de l'effectif. La distance moyenne parcourue au TM6 était de 635,55 \pm 89,38 mètres avec des extrêmes de 441 et 849 mètres. Une proportion de $15 \%$ de nos patients avait parcouru une distance inférieure à la normale. On notait une association statistiquement significative entre le sexe féminin $(\mathrm{p}=0,005)$, le délai diagnostique $(\mathrm{p}=0,006)$ et les distances parcourues anormales. Par ailleurs, $67,3 \%$ des patients avaient un TV à la spirométrie, dominé par le syndrome restrictif $(81,58 \%)$. Comparée à la spirométrie, le TM6 était sensible dans 78,95\% des cas et spécifique dans 2,7\%.

Conclusion. La TP peut altérer significativement la fonction respiratoire des patients. Le TM6 peut constituer un instrument pour l'évaluation fonctionnelle des séquelles de TP.

MOTS CLÉS: Tuberculose; Test de marche; Spirométrie. 


\section{INTRODUCTION}

La tuberculose (TB) constitue un problème majeur de santé publique en raison du fort taux de mortalité et de morbidité. En 2011, 1'Organisation Mondiale de la Santé (OMS) estimait à 8,7 millions le nombre de cas de tuberculose dans le monde, avec 1,4 million de décès dû à la tuberculose [1]. En Afrique, on a estimé à 2,3 millions le nombre de cas de tuberculose avec 254000 décès dû à la tuberculose [1]. Au Togo pour l'année 2011, 2985 cas de tuberculose toutes formes confondues ont été notifiés dont 2092 nouveaux cas de tuberculose pulmonaire confirmée bacteriologiquement $(\mathrm{TPB}+)$ [2].

Cette pathologie peut être pourvoyeuse de nombreuses séquelles en particulier dans sa localisation pulmonaire. Ces séquelles sont multiformes à type de dilatations des bronches (DDB), d'emphysème pulmonaire, de fibrose pulmonaire, de destruction partielle voire totale du poumon, de surinfections récurrentes, réduisent la capacité fonctionnelle des poumons. On assiste donc à une limitation des activités de la vie quotidienne, et une détérioration de la qualité de vie des personnes porteuses de ces séquelles tuberculeuses [3,4].

Paradoxalement, en Afrique où on a l'incidence de la TB est élevée, peu de données sont disponibles sur l'impact de la TB sur la fonction respiratoire. Le test de marche de 6 minutes (TM6) est un test de terrain, validé et couramment utilisé pour évaluer la capacité fonctionnelle à un niveau sous-maximal, et les effets du réentraînement à l'effort des patients cardiaques et pulmonaires [5]. Le but de cette étude était d'évaluer la tolérance à l'effort sous-maximal chez les anciens cas de TPB+ traités et déclarés guéris.

\section{MATERIEL ET METHODES}

\section{Population et type d'étude}

Il s'est agi d'une étude transversale, descriptive et analytique, qui s'est déroulée du 1er Novembre 2012 au 30 Juin 2013 dans le service de pneumologie du CHU Sylvanus Olympio de Lomé. A partir du registre de notification des cas de TB, les nouveaux cas de TPB+ traités et déclarés guéris ont été contactés par téléphone et reçus en consultation de pneumologie pour le recueil des données cliniques. Un TM6 et une spirométrie étaient gratuitement faites à chaque enquêté. Les patients étaient âgés d'au moins 18 ans, et avaient été pris en charge dans un des centres de diagnostic et de traitement (CDT) de la région sanitaire de Lomé commune.

Étaient inclus dans ce travail, tous les patients nouveaux cas $\mathrm{TPB}+$, traités et déclarés guéris au cours des 04 dernières années précédant l'étude (soit du 1er/07/2008 au 31/07/2012) qui avaient données leur consentement éclairé.

Les patients qui avaient présenté des signes d'une rechute tuberculeuse, une pathologie dégénérative neuromusculaire, toutes pathologies cardiovasculaires en cours ou récentes, un asthme ou une bronchite chronique avaient été exclus.

\section{Explorations fonctionnelles respiratoires et défini- tions opérationnelles}

Mesure de la tolérance à un effort sous-maximal

Pour l'évaluation de la capacité fonctionnelle à un niveau sous-maximal, nous avons utilisé le test de marche de six minutes (TM6) selon les recommandations de l'ATS [5]. Des consignes étaient données aux patients. Ils étaient priés d'avoir un habillement confortable, de porter des chaussures appropriées pour marcher, et de n'avoir pas réalisé un exercice intense dans les deux heures précédant le TM6.

Le TM6 était réalisé en plein air, dans un couloir plat de 30 mètres. Le parcours a été marqué chaque 3 mètres. Des instructions avaient été données avant chaque TM6: l'effet, le patient devait marcher le plus rapidement possible, et non courir, afin de parcourir la plus grande distance en six minutes. Toutefois, il pouvait s'arrêter si besoin et reprendre la marche ensuite. Afin de ne pas influencer la vitesse de marche, le patient marchait seul, sans aucun encouragement. Il avait été convenu d'arrêter la marche en cas de désaturation importante en oxygène, de sensation d'asphyxie, d'une douleur musculaire insupportable, de vertiges ou d'une cyanose des extrémités.

La tension artérielle, la saturation périphérique en oxygène $(\mathrm{SpO} 2)$ et la fréquence cardiaque (FC) étaient mesurées avant et immédiatement après la réalisation du TM6. Il en était de même pour la dyspnée qui était évaluée par l'échelle de Borg. La distance parcourue par le patient au TM6 était considérée comme anormale lorsqu'elle était inférieure à limite inférieure de la normale (LIN). Le calcul de la distance théorique (DT), attendue selon le sexe, a été effectué selon les équations d'ENRIGHT and SHERILL [5].

\footnotetext{
Chez l'homme

$\mathrm{DT}=(7.57 \times$ Taille $(\mathrm{cm}))-(5.02 \times$ Age $(\mathrm{ans}))-(1.76 \times$

Poids (kg)) - $309 \mathrm{~m}$

$\mathrm{LIN}=$ distance théorique $-153 \mathrm{~m}$.

Chez la femme

$\mathrm{DT}=(2.11 \times$ Taille $(\mathrm{cm}))-(5.78 \times$ Age $(\mathrm{ans}))-(2.29 \times$

Poids (kg)) - 667m.

LIN $=$ distance théorique $-139 \mathrm{~m}$.

Toute dyspnée $\geq$ à 5/10 à l'échelle de Borg après la
} 
marche a été considérée comme cliniquement anormale et toute dyspnée $\geq$ à $1 / 10$ à l'échelle de Borg au repos (avant le test) a été également considérée comme anormale.

Une $\mathrm{SpO} 2<92 \%$ a été considérée comme cliniquement anormale. Une variation de la saturation $(\mathrm{SpO} 2$ fin - SpO2 début) de plus de 4 points a été retenue pour porter le diagnostic de désaturation lors du TM6.

\section{Mesures spirométriques}

Nous avons utilisé un spiromètre Minispir ${ }^{\circledR}$ avec capteur de débit à turbine à usage unique. Les résultats de la spirométrie étaient calqués sur les critères de l'ATS (American Thoracic Society)/ERS (European Respiratory Society) [6].

- Spirométrie normale lorsque le VEMS/CVF $>70 \%$ des valeurs prédites, le VEMS $\geq 80 \%$ et $C V F \geq 80 \%$.

- Trouble ventilatoire obstructif lorsque le VEMS/ $\mathrm{CVF} \leq 70 \%$ avec $\mathrm{CVF}>80 \%$ des valeurs prédites.

- Trouble ventilatoire restrictif lorsque le VEMS/CVF $>70 \%$ et $\mathrm{CVF}<80 \%$ des valeurs prédites.

- Trouble ventilatoire mixte lorsque le VEMS/CVF < $70 \%$ et la CVF $<80 \%$ des valeurs prédites.

La sévérité du trouble a été classé en fonction du VEMS. Le trouble était

- Léger lorsque le VEMS $\geq 70 \%$.

- Modéré lorsque le $70 \%<$ VEMS $\geq 50 \%$.

- Sévère lorsque le VEMS $<50 \%$.

Pour chaque exercice respiratoire, 3-8 mesures étaient effectuées et la meilleure était retenue. Les patients, qui avaient un trouble ventilatoire, ont été suivis dans le service de pneumologie du CHU Sylvanus Olympio de Lomé (Togo).

\section{Retard diagnostique}

Le retard diagnostique a été défini par un délai diagnostique supérieur à 3 mois.

\section{Analyses des données}

Les données ont été analysées avec le logiciel Epi info version 3.5.1. Le test de Chi2 ou le test exact de Fisher a été utilisé pour la comparaison des variables catégorielles lorsque nécessaire. Les valeurs moyennes ont été présentées avec l'écart type comme indice de dispersion. Le coefficient de corrélation linéaire $r$ de Pearson a servi pour la mesure de corrélation entre les variables quantitatives.

Une régression logistique a permis de tester l'association entre les variables qualitatives dépendantes à deux modalités et, une ou plusieurs autres variables indépendantes qualitatives et/ou quantitatives. Les potentiels facteurs de confusion considérés étaient l'âge, le sexe, la profession, et le tabagisme. Les Odds Ratios (OR) avec leur intervalle de confiance à 95\% ont été déterminés après ajustement sur les facteurs de confusion précités. Les liaisons entre les variables étaient considérées comme statistiquement associées au seuil de 0,05 .

\section{RÉSULTATS}

\section{Taille de la population d'étude}

Au total, 351 patients ont été répertoriés à partir des registres de traitement de la TB. Un effectif de 113 $(32,19 \%)$ d'entre eux a constitué notre échantillon d'étude (Figure 1).

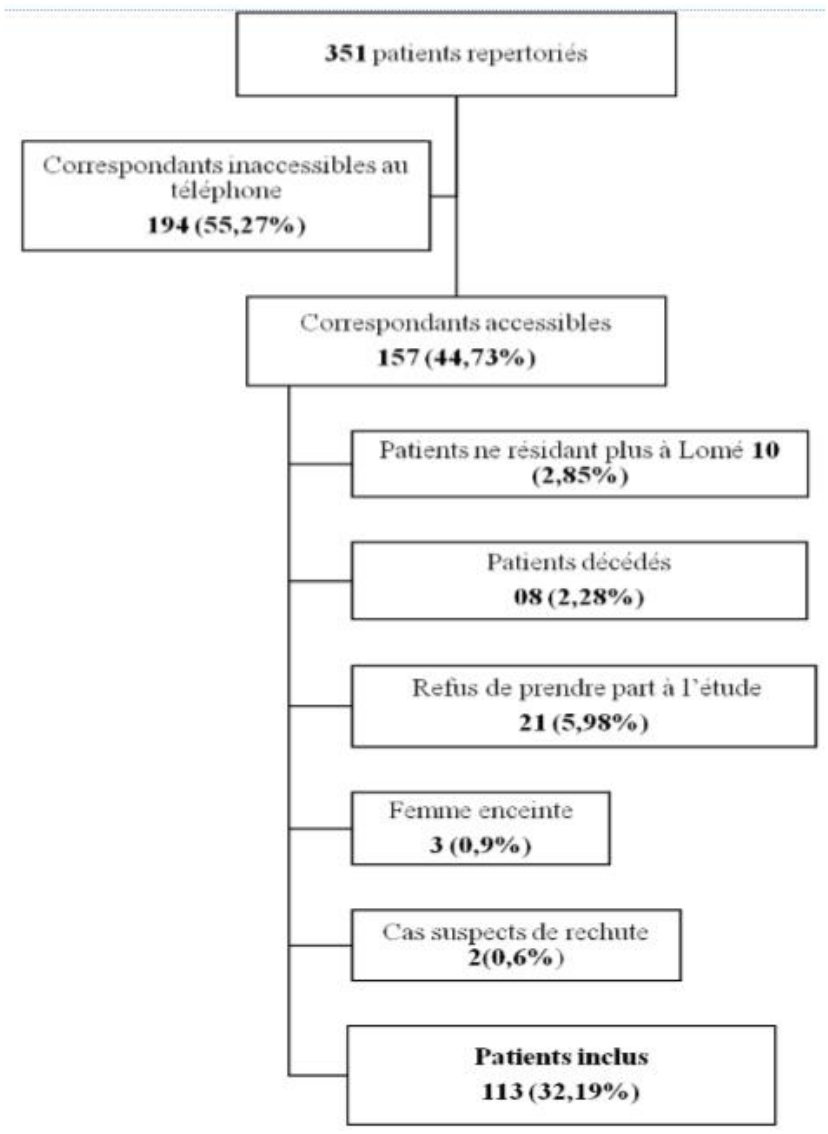

FIGURE 1. Mode de recrutement des patients.

\section{Caractéristiques sociodémographiques}

Nous avons noté une prédominance masculine

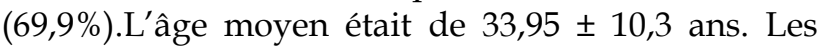
patients âgés de 18 à 45 ans représentaient $88,5 \%$ de l'effectif. La majorité des patients évoluait dans le secteur informel et avait un niveau économique faible $(81,4 \%)$.

Les patients exerçant des professions exposant à des irritantes pulmonaires étaient au nombre de 32 $(28,4 \%)$. La majorité des patients $(93,8 \%)$ avaient été scolarisés et $71,7 \%$ d'entre eux avaient au moins le niveau du secondaire. 


\section{Antécédents}

La prévalence du VIH était de 16,8\%. Le traitement antirétroviral a été initié chez $84,2 \%$ des sujets infectés par le VIH. La prévalence du tabagisme était de 17,7\%. Parmi les patients tabagiques, 75\% étaient sevrés, et $45 \%$ avaient une consommation supérieure à 10 paquets- années.

\section{Délai de mise sous traitement}

Le délai moyen de mise sous traitement (DMST) était de 4,23 $\pm 4,91$ mois. On notait un retard diagnostique chez $40(35,4 \%)$ des patients.

Le délai moyen écoulé entre la fin du traitement anti-

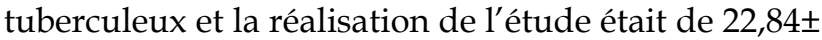
12, 26 mois. Ce délai était supérieur à 12 mois pour $75,2 \%$ des patients.

Distances parcourues en fonction des normes lors du test de marche de 6 minutes (TM6)

La distance parcourue moyenne était de 635,55 \pm 89,38 mètres avec des extrêmes de 441 et 849 mètres.
Parmi nos patients, 17(15\%) d'entre eux avaient parcouru une distance inférieure à limite inférieure à la LIN.

Analyse univariée des caractéristiques des patients selon la distance parcourue au TM6

On notait une association statistiquement significative entre le sexe féminin $(p=0,005)$, le délai diagnostique $(p=0,006)$ et les distances parcourues au TM6. Ainsi les patients ayant un délai diagnostique supérieur à 3 mois avaient une probabilité plus élevée de parcourir une distance inférieure à la LIN (Tableau I).

Analyse multivariée du délai diagnostique et de la qualité de vie selon la distance parcourue au TM6.

Les patients, dont le délai diagnostique supérieur à 3 mois, avaient une probabilité plus grande de parcourir une distance inférieure à LIN au TM6 indépendamment de l'âge, du sexe, de la notion de tabagisme et de l'exposition à une profession à risque (OR ajusté $=3,6[1,2 ; 11,5]$ ) (Tableau 2).

\section{TABLEAU 1 Analyse univariée des caractéristiques des patients selon le TM}

\begin{tabular}{|c|c|c|c|c|c|}
\hline Variables & $\begin{array}{c}\text { Normale } \\
\text { N (\%) }\end{array}$ & $\begin{array}{l}\text { TM6 } \\
\text { Anormale } \\
\text { N (\%) }\end{array}$ & Total & OR [IC 95\%] & $p$-value \\
\hline \multicolumn{6}{|l|}{ Sexe } \\
\hline $\mathrm{M}$ & $72(91,1)$ & $7(8,9)$ & 79 & \multirow{2}{*}{$4,3[1,5 ; 12,5]$} & \multirow{2}{*}{0,005} \\
\hline $\mathrm{F}$ & $24(70,6)$ & $10(29,4)$ & 34 & & \\
\hline \multicolumn{6}{|l|}{ Professions } \\
\hline Non exposées & $68(84,0)$ & $13(16,0)$ & 81 & $0,75[0,2 ; 2,7]$ & \multirow{2}{*}{0,44} \\
\hline Exposées & $28(87,5)$ & $4(12,5)$ & 32 & & \\
\hline \multicolumn{6}{|l|}{ Tabagisme } \\
\hline Non & $79(84,9)$ & $14(15,1)$ & 93 & $0,99[0,2 ; 4,2]$ & \multirow{2}{*}{0,62} \\
\hline Oui & $17(85,0)$ & $3(15,0)$ & 20 & & \\
\hline \multicolumn{6}{|l|}{ VIH } \\
\hline négatif & $82(87,2)$ & $12(12,8)$ & 94 & $2,4[0,6 ; 8,9]$ & \multirow{2}{*}{0,13} \\
\hline positif & $14(73,7)$ & $5(26,3)$ & 19 & & \\
\hline \multicolumn{6}{|l|}{ Durée traitement } \\
\hline 6 mois & $66(84,6)$ & $12(15,4)$ & 78 & $0,9[0,3 ; 2,8]$ & \multirow{2}{*}{0,88} \\
\hline 8 mois & $30(85,7)$ & $5(14,3)$ & 35 & & \\
\hline \multicolumn{6}{|c|}{ Délai fin traitement-test } \\
\hline$\leq 12$ mois & $23(82,1)$ & $5(17,9)$ & 28 & $0,76[0,2 ; 2,4]$ & \multirow{2}{*}{0,63} \\
\hline$>12$ mois & $73(85,9)$ & $12(14,1)$ & 85 & & \\
\hline \multicolumn{6}{|l|}{ Délai diagnostique } \\
\hline$\leq 3$ mois & $67(98,8)$ & $6(8,2)$ & 73 & $4,2[1,4 ; 12,5]$ & \multirow{2}{*}{0,006} \\
\hline$>3$ mois & $29(72,5)$ & $11(27,5)$ & 40 & & \\
\hline
\end{tabular}




\section{Variation de la $\mathrm{SpO} 2$}

Une désaturation en oxygène a été notée chez $42,5 \%$ des patients. Elle variait de 1 à $5 \%$. Seulement 1,8\% patients avaient une désaturation significative $(\geq 4 \%)$.

\section{Mesures Spirométriques}

La spirométrie était pathologique dans $67,3 \%$ des cas. Ces troubles ventilatoires étaient dominés parle syndrome restrictif $(81,58 \%)$ (Tableau 3$)$.

\section{TABLEAU 2 Répartition des patients selon les conclusions à la spirométrie}

\begin{tabular}{|c|c|c|c|c|}
\hline Conclusion à la Spirométrie & Effectif & $\begin{array}{c}(\%) / \text { au sein } \\
\text { des troubles } \\
\text { ventilatoires }\end{array}$ & $\begin{array}{c}(\%) / \text { aux } \\
\text { troubles } \\
\text { ventilatoires }\end{array}$ & 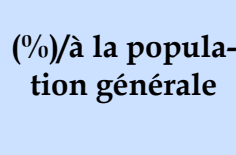 \\
\hline Spirométrie normale & 37 & - & - & 32,7 \\
\hline Troubles ventilatoires & 76 & - & 100 & 67,3 \\
\hline Restrictifs & 62 & 100 & 81,58 & 54,9 \\
\hline Légère & 36 & 58,1 & 47,37 & 31,85 \\
\hline Modérée & 23 & 37,1 & 30,26 & 20,35 \\
\hline Sévère & 3 & 4,8 & 3,95 & 2,7 \\
\hline Obstructifs & 7 & 100 & 9,21 & 6,2 \\
\hline Légère & 6 & 85,7 & 7,9 & 5,3 \\
\hline Modérée & 0 & 0 & 0 & 0 \\
\hline Sévère & 1 & 14,3 & 1,31 & 0,9 \\
\hline Mixtes & 7 & 100 & 9,21 & 6,2 \\
\hline Légère & 0 & 0 & 0 & 0 \\
\hline Modérée & 3 & 42,9 & 3,95 & 2,7 \\
\hline Sévère & 4 & 57,1 & 5,26 & 3,5 \\
\hline
\end{tabular}

\section{TABLEAU 3}

Analyse multivariée du délai diagnostique et du TM6 en fonction des conclusions spirométriques

\begin{tabular}{|c|c|c|c|c|c|}
\hline Variables & Effectifs & $\begin{array}{c}\text { Troubles } \\
\text { ventilatoires } \\
\mathbf{N}(\%)\end{array}$ & $\begin{array}{l}\text { OR brut } \\
\text { [IC95\%] }\end{array}$ & $\begin{array}{c}\text { OR ajusté } \\
\text { [IC 95\%] }\end{array}$ & VR \\
\hline \multicolumn{6}{|c|}{ Délai diagnostique } \\
\hline$\leq 3$ mois & 73 & $44(60,3)$ & & & \\
\hline$>3$ mois & 40 & $32(80,0)$ & $2,6[1,1 ; 6,5]$ & $2,8[1,1 ; 7,0]$ & 7,14 \\
\hline \multicolumn{6}{|l|}{ Test de marche } \\
\hline Normal & 96 & $60(62,5)$ & & & \\
\hline Anormal & 17 & $16(94,1)$ & $9,6[1,2 ; 75,4]$ & $8,9[1,1 ; 73,2]$ & 7,86 \\
\hline
\end{tabular}




\section{Analyse multivariée du délai diagnostique et du TM6 en fonction des conclusions spirométriques.}

Les patients qui avaient un délai diagnostique supérieur à 3 mois, et ceux qui avaient parcourue une distance inférieure à LIN au TM6, avaient une probabilité plus grande d'avoir des troubles ventilatoires à la spirométrie. Et ce indépendamment de l'âge, du sexe, de la notion de tabagisme et de l'exposition à une profession à risque (respectivement $\mathrm{OR}=2,8$; $\mathrm{OR}=8,9)($ Tableau 3$)$.

\section{Evaluation de la performance diagnostique du TM6 comparée à la spirométrie}

La sensibilité (Se) était de $78,95 \%$ et la spécificité (Sp) de 2,7\%. Quant à la valeur prédictive positive (VPP) elle était de $62,5 \%$ et de $5,9 \%$ pour la valeur prédictive négative (VPN).

\section{DISCUSSION}

Le TM6 reflète la capacité fonctionnelle respiratoire chez un individu au cours de ces activités de quotidiennes. Dans notre étude, on notait une limitation à l'effort chez $15 \%$ des patients. La distance parcourue moyenne était de 635,55 $\pm 89,38 \mathrm{~m}$. Di Naso au Brésil et Ando au Japon retrouvaient respectivement une distance parcourue moyenne moindre de 484,21 \pm $74,01 \mathrm{~m}$ et de $342 \pm 77 \mathrm{~m}[3,7]$. Cette différence avec nos résultats pourrait s'expliquer par le fait que les patients de Ando était âgés (moyenne d'âge de 71 ans) et avaient des antécédents de chirurgie thoracique ou des séquelles sévères de pleurésie remontant à plus de 30 à 40 années auparavant. Également dans l'étude de Di Naso, 60\% des patients étaient tabagiques. Tous ceux-ci pourraient limiter la distance parcourue au TM6.

Notre population d'étude était majoritairement jeune avec une prédominance masculine. Classiquement la tuberculose touche plus les sujets jeunes et de sexe masculin, qui sont économiquement productifs. Ainsi les incapacités fonctionnelles que pourraient entrainer la tuberculose pourraient avoir des conséquences sur les activités professionnelles de ces patients avec une répercussion sur leur revenu. D'autant plus que la majorité de nos patients évoluait dans le secteur informel et avait probablement un faible revenu. Ceci pourrait constituer un frein pour la prise en charge d'éventuelles séquelles occasionnées par la tuberculose. En effet en dehors des antituberculeux qui sont gratuits, tous les autres frais occasionnés par la tuberculose sont à la charge des patients.

On notait également une association statistiquement significative entre le délai diagnostique et le TM6. Ainsi les patients qui avaient un délai diagnostique . supérieur à 3 mois avaient une probabilité plus élevée de parcourir une distance inférieure à la LIN. Ceci nous pousse à affirmer qu'un diagnostic et une mise sous traitement précoces permettront de réduire l'impact à long terme de la tuberculose pulmonaire sur la fonction respiratoire.

Les patients tabagiques étaient au nombre de 20 $(17,7)$ et $45 \%$ avaient une consommation supérieure à 10 paquets-années. L'association tabac et séquelles de tuberculose pourrait être doublement délétères et péjoratif pour la fonction respiratoire. Won Lee et al. en Corée du Sud a noté qu'il existait une association entre les patients qui avaient un antécédent de tuberculose pulmonaire et la survenue de maladie pulmonaire obstructive [8].

Lam et al. en République Populaire de Chine retrouvait chez les patients tabagiques à plus de vingt paquets-années et qui avaient d'importantes séquelles de tuberculose à la radiographie, une prévalence élevée d'obstruction des voies aériennes [9]. Pour Lam et al. le tabac et les séquelles de tuberculoses ont un effet délétère additif et non synergique sur la fonction pulmonaire [10]. Ces observations soulignent la nécessité d'intégrer un programme d'aide au sevrage tabagique dans les interventions antituberculeuses.

Nous avons noté également dans notre étude, une association statistiquement significative entre le TM6 et les résultats de la spirométrie. Ainsi on observait significativement plus de troubles ventilatoires chez les patients ayant parcourus une distance inférieure à LIN au TM6 que ceux ayant parcouru une distance dans les normes. Nous pouvons dire qu'en l'absence de spirométrie, le TM6 pourrait permettre de détecter une altération de la fonction respiratoire. Mais cependant une prudence s'impose, car l'évaluation de la performance diagnostique du TM6 comparée à la spirométrie montre une sensibilité de $78,95 \%$ et une spécificité de $2,7 \%$. La valeur prédictive positive était de $62,5 \%$ et la valeur prédictive négative de $5,9 \%$. On en déduit que le TM6 n'est pas un très bon instrument de diagnostic, mais néanmoins il peut constituer un instrument de "dépistage" dans les endroits où la spirométrie n'est pas accessible.

Le TM6 apparait également comme un bon outil de monitorage pour l'évaluation de la dyspnée lors des programmes de réhabilitation fonctionnelle respiratoire. En effet ces programmes de réhabilitations, très en vogue dans la BPCO apparait comme modalité thérapeutique chez les patients avec séquelles de tuberculose pulmonaire et handicapés par la dyspnée. Dans l'étude d'Ando, on notait une amélioration de la distance parcourue de 64 mètres après un programme de réhabilitation de 6 mois [7]. 


\section{CONCLUSION}

Cette étude fournit une évaluation multidimensionnelle de l'impact de la tuberculose pulmonaire sur la fonction pulmonaire et la tolérance à l'effort sous maximal. Nos résultats représentent une estimation

\section{CONFLIT D'INTÉRÊTS}

Aucun.

\section{RÉFÉRENCES}

1. World Health Organization. WHO report 2012.Global tuberculosis control. 2012: 258.

2. Programme National de Lutte contre la Tuberculose. Rapport annuel des activités de lutte contre la tuberculose au Togo en 2011 . PNLT 2012 :58.

3. Di Naso F C, Pereira J S, Schuh S J. Functional evaluation in patients with pulmonary tuberculosis sequelae. Rev Port Pneumol. 2011; 17 (5) : 216-21.

4. Long R, Maycher B, Dhar A. Pulmonary tuberculosis treated with directly observed therapy: serial changes in lung structure and function. Chest 1998; 113(4): 933-43.

5. ATS Statement: Guidelines for the six-minute walk test. Am J Respir Crit Care Med 2002; 166:111117. prudente de la vraie nature et de l'étendue des séquelles fonctionnelles pulmonaires liées à la tuberculose pulmonaire. Le TM6 peut constituer un instrument pour l'évaluation fonctionnelle des séquelles liées à cette pathologie dans les régions ou la spirometrie n'est pas disponible.
6. Pellegrino R, Viegi G, Brusasco V. Stratégies d'interprétation des explorations fonctionnelles respiratoires. Rev Mal Respir 2006 ; 23 : 17S79-17S104. Traduction de «Interpretative strategies for lung function tests ». Eur Respir J 2005 ; 26 : 948-968. Série du groupe de travail ATS/ERS.

7. Ando M, Mori A, Esaki H. The effect of pulmonary rehabilitation in patients with post-tuberculosis lung disorder. Chest 2003; 123; 1988-95.

8. Ait-khaleb N, Enarson D. Tuberculose, manuel pour les étudiants en médecine, WHO/CDS/TB/1999.272.

9. Dakin J et coll. Maîtriser les EFR de la théorie à la clinique. Elsevier Masson. Paris. 2007.

10. Beigelman C, Brauner M. Quelles attitudes adopter devant des séquelles de tuberculose mises en évidence sur une radiographie thoracique ? Rev Mal Respir 2004 ; 21: 3S60-3S4. 